\title{
3 Research Square

\section{Transcriptional profiling reveals molecular basis and the role of arginine in response to low-pH stress in Pichia kudriavzevii}

Hao Ji ( $20180031 @ w z u . e d u . c n)$

Wenzhou University https://orcid.org/0000-0003-2371-6098

Xiameng Dong

Wenzhou Vocational College of Science and Technology

Kailun Zhang

Wenzhou University

Libo Jin

Wenzhou University

Renyi Peng

Wenzhou University

sue Lin

Wenzhou University

Da Sun ( $\nabla$ sunday@wzu.edu.cn )

Wenzhou University

KeXu ( $\nabla$ godxu1987@163.com)

Wenzhou University

\section{Research}

Keywords: Pichia kudriavzevii, low-pH, RNA-Seq, arginine, cell aggregation

Posted Date: December 13th, 2019

DOI: https://doi.org/10.21203/rs.2.18639/v1

License: (c) (1) This work is licensed under a Creative Commons Attribution 4.0 International License. Read Full License

Version of Record: A version of this preprint was published at Journal of Bioscience and Bioengineering on December 1st, 2020. See the published version at https://doi.org/10.1016/j.jbiosc.2020.07.014. 


\section{Abstract}

\section{Background}

The non-conventional yeast Pichia kudriavzevii possesses a unique ability to tolerate various environmental stresses particularly low-pH stress. Thus, it is considered to be a promising biotechnological host for the production of various organic acids under low-pH conditions. However, little is known about the low-pH stress response in P. kudriavzevii, which significantly restricts its future development.

\section{Results}

In this study, P. kudriavzevii JLY1107 showed great tolerance to low-pH stress, but its cell aggregation upon acidic conditions is unfavorable for the development of low-pH fermentation. To explore the molecular basis, we conducted RNA-Seq to compare global gene expression in response to low-pH. Among the 429 differentially expressed genes, the genes associated with regulation of membrane lipid composition, filamentous growth and arginine metabolism were selected for in-depth discussions. The up-regulation of genes associated with arginine uptake and degradation suggests a potential role of arginine in response to low-pH strsss. We therefore present data supporting the hypothesis that $P$. kudriavzevii maintains intracellular homeostasis by using the ammonia produced by arginine catabolism. Furthermore, external addition of arginine significantly enhances growth and reduces cell aggregation of P. kudriavzevii under low-pH conditions.

\section{Conclusions}

Arginine was demonstrated to be a promising molecule for improving cell growth and preventing cell aggregation under extremely low-pH conditions. Our study is a step towards developing the nonconventional yeast $P$. kudriavzevii as a platform host for the production of organic acids under low-pH conditions.

\section{Background}

Microbial fermentation is a promising approach for obtaining organic acids from renewable resources that constitute a key group among top platform chemicals [1]. Various types of microorganisms have been developed for this purpose and in-depth knowledge of the production processes has been obtained, but the industrially large-scale production still has many challenges. Since the $\mathrm{pH}$ of fermentation broth is expected to decrease to around 2.0 for acid titers of $50 \mathrm{~g} / \mathrm{L}$ [1], the major relevant bottleneck in the production processes is likely relate to the inhibitory effects of low-pH of the culture broth on cell growth, cell viability and in turn on organic acids accumulation. The use of naturally low-pH tolerant organisms such as yeasts is an alternative route to produce organic acids by microbial fermentation [2]. These strains can be cultivated at low-pH conditions below the $\mathrm{pKa}$ of organic acid, facilitating the downstream 
processing and reducing the cost in $\mathrm{pH}$ titrant. Moreover, the low-pH yeast fermentation with direct crystallization has been shown to be the most attractive from an environmental perspective [3].

While the model yeast Saccharomyces cerevisiae are extensively studied, non-Saccharomyces may be superior in the low-pH production of organic acids. The non-conventional yeast Pichia kudriavzevii (also known as Issatchenkia orientalis or Candida krusei) has emerged as a robust organism with multi-stress tolerance to extreme low-pH, hyperosmotic, and high temperatures [4,5]. Owing to its extremely robust physiology, P. kudriavzevii has been explored for the production of organic acids such as succinic acid, lactic acid, D-xylonate, and it showed excellent fermentation efficiency under the low-pH conditions[6-9], which makes it a good candidate for industrial application. However, the studies for low$\mathrm{pH}$ response in $P$. kudriavzevii are very limited, which significantly restricts the future development of this promising platform microorganism.

The response mechanisms of acid stress have been widely investigated in the model yeast Saccharomyces cerevisiae [10]. However, the obtained knowledge cannot be applied easily to other yeast species. It was previously reported that IoGAS1 encoding a glycosylphosphatidylinositol-anchored protein represents a novel $\mathrm{pH}$-regulated system required for the adaptation of $P$. kudriavzevii to environments $\mathrm{pH}$ [11]. However, more systematic studies underlying low-pH response have not yet been reported. In this study, we focused on P. kudriavzevii JLY1107, a strain isolated from Kazakh cheese that showed high tolerance and morphological differentiation under extremely low-pH conditions. To explore the molecular basis, high-throughput RNA sequencing (RNA-Seq) was conducted to analysis the transcriptome profile of $P$. kudriavzevii in response to extremely low-pH stress. The results may provide more valuable genetic information for the engineering of $P$. kudriavzevii as a platform microorganism for the low-pH fermentation of organic acids.

\section{Results And Discussion}

\section{Cell growth of $P$. kudriavzevii under low-pH condition}

P. kudriavzevii previously showed greater tolerance to low-pH stress than some other yeasts such as $S$. cerevisiae and K. lactis [9]. In this study, the strain JLY1107 isolated from Kazakh cheese also exhibited better cell growth than S. cerevisiae W303, especially at pH conditions blew 3.0 (Fig. 1a). P. kudriavzevii grew well at the extremely acidic conditions (down to $\mathrm{pH} 1.5$ ), and its $\mathrm{DCW}$ at $\mathrm{pH} 2.0$ reached $60 \%$ of that at $\mathrm{pH}$ 5.0. Remarkably, P. kudriavzevii exhibited filamentation growth and flocculation under the extremely acidic conditions in both YPD and minimal medium (Fig. 1b). It grew as large aggregates in liquid medium at $\mathrm{pH} 2.0$, forming extensive pseudohyphae (Fig. 1c). These results indicated that low-pH stress induced morphological differentiation in P. kudriavzevii. Flocculation was proved to be a cheap and relatively easy way of cell separation from fermented products upon completion of fermentation $[12,13]$. However, flocculation occurring during the fermentation process is thought to be a limiting factor, since it really affects the transfer of nutrients and diffusion of oxygen, resulting in early termination of fermentation $[14,15]$. Thus, we believe that the cell aggregation of $P$. kudriavzevii upon acidic conditions 
is unfavorable for the development of organicacid low-pH fermentation or fermentation without $\mathrm{pH}$ control by using this acid-tolerant host. For $S$. cerevisiae strains, flocculation occurs over a wide $\mathrm{pH}$ range of 2.5 to 9.0 [12], while it occurs under the $\mathrm{pH}$ values lower than 2.5 in P. kudriavzevii. In addition, Candida albicans and Yarrowia lipolytica grow mainly in the form of yeast under acidic environment, and mainly in the form of mycelia when the environment is neutral or alkaline $[16,17]$. These results suggest a different response mechanism to low-pH stress in P. kudriavzevii.

\section{Statistics and quality assessment of the RNA sequencing data}

To explore the molecular basis, we conducted RNA-Seq to compare global gene expression in $P$. kudriavzevii at $\mathrm{pH} 2.0$ and 5.5, respectively. The draft genome sequence of $P$. kudriavzevii CBS573 reported by Douglass et al was used as reference [18]. Transcriptome data was deposited in the National Center for Biotechnology Information database with an accession number of PRJNA556936. As shown in Additional file 1: Table S2, after removal of adaptors, ambiguous reads and low-quality reads, we obtained an average of 22.4 million total clean reads of approximately $6.7 \mathrm{~Gb}$ for each sample. The average of GC contents was $41.5 \%$, and the Q 30 was more than $95.0 \%$ of the reads. Approximately 43 million reads from each sample were mapped to the reference genome, accounting for more than $95 \%$ of the total reads (Additional file 1:Table S3). The mapped reads were then spliced using String Tie and aligned to the reference genes.

\section{Transcriptional response of $P$. kudriavzevii under low-pH condition}

Expression levels of 5149 genes in each sample were calculated using the FPKM method. The Pearson's correlation coefficients among biological replicates are all greater than 0.87 , suggesting a good reproducibility of the experiments (Additional file 1: Figure S1). The comparative transcriptomic analysis was then performed to screen DEGs. As shown in Fig. 2, 429 genes were identified as DEGs by choosing the cut-off value (fold change $\geq 2$ and FDR $<0.05$ ) in DE-seq R Package, of which 309 genes were significantly upregulated and 120 genes were significantly downregulated at $\mathrm{pH} 2.0$ condition. To verify the quality of the RNA-Seq data, qRT-PCR analysis was conducted on 10 randomly selected genes. The strong correlation between the RNA-Seq and qRT-PCR results indicated high reliability of our transcriptomic profiling data (Additional file 1: Figure S2).

All of the DEGs successfully matched with entries in at least one of the databases including COG, GO, KEGG, KOG, NR, Pfam, Swiss-Port and eggNOG databases. Based on Gene Ontology (GO) analysis, 93\% of DEGs (399 out of 429) were categorized into several functional groups (Fig. 3a). The commonly upregulated genes were involved in biological processes including cellular amino acid metabolic process (GO: 0006520), transmembrane transport (GO: 0055085), cellular response to stimulus (GO: 0051716), primary metabolic process (GO: 0044238) and filamentous growth (GO: 0030447), whereas downregulated genes were implicated in cellular component organization or biogenesis (GO: 0071840) and carbohydrate metabolic process (GO: 0005975). Additionally, statistical analysis of the metabolic pathways involving the DEGs was performed using KEGG pathway mapping. We concluded that 80 pathways were significantly enriched, particularly biosynthesis of antibiotics, biosynthesis of amino 
acids, carbon metabolism, ribosome biogenesis in eukaryotes and purine metabolism were the five most highly enriched pathways (Fig. 3b).

The high enrichment of steroid biosynthesis, biosynthesis of unsaturated fatty acids, fatty acid metabolism and glycerophospholipid metabolism implied that $P$. kudriavzevii altered the membrane lipid composition in response to extremely low-pH stress. Actually, controlling membrane lipid composition has been considered to play a key role in multiple stresses tolerance [19]. Membrane engineering via incorporation of trans unsaturated fatty acids enabled increased tolerance in Escherichia coli to a variety of adverse conditions such as low-pH and high temperature [20]. Studies in Candida glabrata and Debaryomyces hansenii also highlighted the role of membrane lipid composition in response and tolerance to low-pH stress [21-23].

Among the DEGs involved in filamentous growth, a gene enconding Flo11 domain-containing protein (Fdc) showed very high downregulation ( $\log _{2}$ fold change $=-7.59$ ) under the condition of $\mathrm{pH} 2.0$, implying that it might act as a negative regulator of morphological differentiation in $P$. kudriavzevii. It is reported the Flo adhesin protein family are required for the interactions of fungal cells with each other in the processes of flocculation and filamentation [24]. Flo11 as a cell surface flocculin is one of the most intensively studied adhesion molecules, and its expression is responsible for the morphological changes [25]. We analyzed the structural of the Fdc by using the online tool of Pfam (http://pfam.xfam.org/). Besides Flo11 domain, there are two fungal cellulose binding domains in the C-terminal of $\mathrm{Fdc}$, which is quite different from a typical Flo11 protein (Additional file 1: Figure S3), hence further studies on its molecular function are needed.

\section{Transcriptional profiling reveals the potential role of arginine metabolism}

Regulation of basic amino acid metabolism is also remarkable in the transcriptome data, particularly for arginine (Fig. 4a). CAN1 encoding an arginine permease was reported as a more effective transporter for arginine uptake in S. cerevisiae [26]. In this study, the expression of CAN1 was up-regulated in response to extremely low-pH stress, implying a great demand for arginine in intracellular microenvironment. CAR1 encoding arginase and DUR1 encoding urea amidolyase are also up-regulated, suggests that more amount of ammonia might be produced in low-pH conditions to neutralize the intracellular protons, and that is favorable for maintaining the $\mathrm{pH}$ homeostasis. Considering the key role of arginase in arginine catabolism and its significant changes in transcription levels, we measured the specific activity of arginase of $P$. kudriavzevii cultured at different $\mathrm{pH}$ conditions. Compared with culturing at $\mathrm{pH} 5.5$, the enzyme activity increased by $85.3 \%$ at the condition of pH 2.0 (Fig. $4 \mathrm{~b}$ ), while the intracellular ammonia concentration increased by $44.6 \%$ (Fig. 4c). These results confirmed the above hypothesis and suggested that arginine catabolism via arginase (Car1) and urea amidolyase (Dur1) might play a key role in the tolerance to extreme low-pH stress in P. kudriavzevii. Conversely, a study in Candida ablicans indicated that it is alkaline rather than the acidic conditions inducing arginine uptake via Can 1 and degradation via Car1 [16]. Arginine has also been reported to be involved in the acid tolerance of $E$. coli [27]. However, unlike the results from our study, arginine-dependent acid resistance system in $E$. coli is comprised of the 
cytoplasmic inducible arginine decarboxylase (AdiA) and the inner membrane arginine/agmatine antiporter (AdiC), that provide robust protection against extremely acid stress by decarboxylation reaction $[27,28]$.

\section{Arginine addition enhances growth an $\mathrm{d}$ reduces cell aggregation under low-pH conditions}

To further evaluate the potential effects of arginine, $5 \mathrm{mM}$ arginine was added to the YPD medium at $\mathrm{pH}$ 2.0 or 5.5. The results showed that extracellular addition of $5 \mathrm{mM}$ arginine significantly enhanced the growth of $P$. kudriavzevii at pH 2.0 (Fig. 5a). Meanwhile, the pHi was improved in the present of arginine (Fig. 5b). Arginine has been reported to act as a compatible solute to protect Candida glabrata against hyperosmotic stress [29], and accumulate intracellularly to protect cells from ethanol damage [30]. Our results further confirmed the positive role of arginine on protecting $P$. kudriavzevii against low-pH stress.

Remarkably, the cell aggregation exhibited strongly reduction in the present of $5 \mathrm{mM}$ arginine (Fig. 6), that is favorable for organic acid fermentation at low-pH conditions or without $\mathrm{pH}$ control. This result is not entirely unexpected, since it has been reported that the availability of ammonium or specific amino acids modulates morphological differentiation [31, 32]. With the addition of arginine, increased concentration of intracellular ammonia may regulate the expression of genes involving in cell aggregation such as FLO11. As stated earlier, the function of Flo11 domain-containing protein is not clear, therefore more genetic studies are required for the deeper research on the regulatory mechanism. Recently, an genetic toolkit based on CRISPR/Cas9 system was developed in P. kudriavzevii [33] , which makes gene manipulation more efficient and convenient.

\section{Conclusion}

In this study, we provide insights into the molecular basis of low-pH response in $P$. kudriavzevii, which was previously unknown. By using transcriptomics analysis, we observed that the genes associated with different biological processes including regulation of membrane lipid composition, filamentous growth and regulation of basic amino acid metabolism were differentially regulated. Furthermore, we demonstrate for the first time that in $P$. kudriavzevii, arginine might be a promising molecule for improving cell growth and preventing cell aggregate under extremely low-pH conditions. Our study is a step towards developing the non-conventional yeast $P$. kudriavzevii as a platform host for the production of organic acids under low-pH conditions.

\section{Materials And Methods}

\section{Yeast strains, media, and culture conditions}

P. kudriavzevii JLY1107 isolated from Kazakh cheese and S. cerevisiae W303 were maintained as streaks on YPD agar or in $15 \% \mathrm{v} / \mathrm{v}$ glycerol at $-80^{\circ} \mathrm{C}$. All the yeast strains were grown in YPD medium $(2 \%$ glucose, $2 \%$ peptone, $1 \%$ yeast extract, $2 \%$ agar) or yeast nitrogen base (YNB) $(0.67 \%$ yeast nitrogen 
base, $2 \%$ glucose) medium supplemented with essential nutrients and incubated at $30^{\circ} \mathrm{C}$ with shaking at $200 \mathrm{rpm}$. The pH value of the medium was adjusted according to the needs of the experiments.

\section{RNA extraction, library preparation and sequencing}

Log-phase cells were inoculated into YPD medium at pH 2.0 and 5.5 for $4 \mathrm{~h}$. The yeast cells were then harvested and washed twice with precooled PBS buffer. Samples were frozen with liquid nitrogen and stored at $-80^{\circ} \mathrm{C}$ until RNA extraction. Total RNA from each sample was isolated using TRIzol (Ambion, USA). RNA quantity and quality were determined using a Qubit RNA Assay Kit in a Qubit 2.0 Flurometer (Life Technologies, CA, USA), and the NanoPhotometer® spectrophotometer (IMPLEN, CA, USA). RNA integrity and purity were evaluated using the RNA Nano 6000 Assay Kit with Agilent Bioanalyzer 2100 system (Agilent Technologies, CA, USA).

The RNA samples were then sent to BioMarker Biotechnology Corporation (Beijing, China) for construction of cDNA libraries and illumina sequencing. Briefly, a total amount of $1 \mu \mathrm{g}$ RNA per sample was used as input material to construct the libraries using NEBNext Ultra RNA Library Prep Kit (NEB, USA). Hiseq 2500 platform (Illumina, USA) was used to perform the sequencing using a pair-end strategy.

\section{Quality control and read mapping}

Clean data were obtained by discarding the reads containing adaptor, poly-N and low-quality reads from raw data. Q20, Q30, GC-content and sequence duplication level of the clean data were calculated. Clean reads were then mapped to the reference genome (NCBI Accession Number: ASM305444v1) using HISAT2 (http://ccb.jhu.edu/software/hisat2/index.shtml).

\section{Annotation of differentially Expressed genes}

Gene expression levels were calculated by FPKM (Fragments Per Kilobase of transcript per Million mapped reads) using RSEM (RNA-Seq by Expectation Maximization) module provided within Trinity package. To screen the differentially expressed genes (DEGs), a criterion with a false discovery rate (FDR) $\leq 0.05$ and the absolute value of the $\log 2$ ratio $\geq 1$ was used as the threshold to judge the significance of differential gene expression.

DEGs encoding proteins were used for sequence similarity search against protein databases such as $\mathrm{Nr}$ (NCBI non-redundant protein sequence), GO (Gene Ontology), KOG/COG (euKaryotic Ortholog Groups/Clusters of Orthologous Groups), Pfam (Protein family), Swiss-Prot (high quality annotated and non-redundant protein sequence database) and KEGG (Kyoto Encyclopedia of Genes and Genomes), by using Blast2GO software with an E-value threshold of $10^{-5}$ [34].

\section{Enrichment analyses of DEGs}

GO enrichment analysis of DEGs was implemented by the GOseq R packages based Wallenius noncentral hyper-geometric distribution [35]. KOBAS software was used to test the statistical enrichment of 
differential expression genes in KEGG pathways [36].

\section{Real-Time Quantitative PCR (qRT-PCR) validation}

Ten DEGs were randomly selected for the validation of RNA-Seq results by qRT-PCR. Specific primers were designed by Beacon designer software version 8.0, which are list in Additional file 1: Table S1. Actin1 was employed as an internal reference gene. qRT-PCR was performed using SYBR Premix Ex Taq (Takara Bio) on the Light Cycler 480 (Roche Diagnostics, Germany). The amplification program was set to $95^{\circ} \mathrm{C}$ for 30 s and 40 cycles of $95^{\circ} \mathrm{C}$ for $5 \mathrm{~s}, 60^{\circ} \mathrm{C}$ for $20 \mathrm{~s}, 72^{\circ} \mathrm{C}$ for $30 \mathrm{~s}$. Additionally, a thermal denaturing step was used to generate the melting curves to verify the specificity. All the reactions were performed in triplicate biological replicates.

\section{Analytical methods}

Culture broth was centrifuged for $10 \mathrm{~min}$ at $10000 \times \mathrm{g}$. Subsequently, the supernatant was eliminated and yeast cells were dried to constant weight in a drying oven at $105^{\circ} \mathrm{C}$, and then the dry weight of cells (DCW) were calculate.

Yeast cells were collected and washed twice by pre-cooling 0.2 M PBS buffer ( $\mathrm{pH} 7.5)$, and then resuspended for 10 min ice-bath ultrasonic treatment. After centrifuged at $10000 \times \mathrm{g}$ for $10 \mathrm{~min}$ at $4{ }^{\circ} \mathrm{C}$, the supernatant was collected for the detection of enzyme activity and intracellular ammonia concentration. Specific arginase activity was measured by the method described in [37]. Intracellular ammonia concentration was assayed according to the instruction of an ammonia colorimetric assay kit (Megazyme, Ireland). Intracellular pH (pHi) was determined as previously described [38].

\section{Declarations}

\section{Availability of data and materials}

The RNA-seq datasets are available from the National Center for Biotechnology Information database. Other datasets used during the current study are available from the corresponding author on reasonable request.

\section{Acknowledgements}

Not applicable.

\section{Funding}

This work was supported by the National Natural Science Foundation of China (81802251), Natural Science Foundation of Zhejiang Province (LQ20C200015, LQ18H150003).

\section{Contributions}


$\mathrm{HJ}, \mathrm{LJ}, \mathrm{KZ}$, and RP performed the experiments and drafted the manuscript, XD and DS assisted in data presentation and supervised the study, $\mathrm{JH}$ and $\mathrm{KX}$ supervised the study and wrote the manuscript. All authors read and approved the final manuscript.

\section{Corresponding authors}

Correspondence to Hao Ji, Da Sun or Ke Xu.

\section{Ethics declarations}

\section{Ethics approval and consent to participate}

Not applicable.

\section{Consent for publication}

Not applicable.

\section{Competing interests}

The authors declare that they have no competing financial interests.

\section{Additional information}

\section{Publisher's Note}

Springer Nature remains neutral with regard to jurisdictional claims in published maps and institutional affiliations.

\section{Supplementary information}

Additional file 1. Additional figures and tables.

\section{Rights and permissions}

Open Access This article is distributed under the terms of the Creative Commons Attribution 4.0 International License (http://creativecommons.org/licenses/by/4.0/), which permits unrestricted use, distribution, and reproduction in any medium, provided you give appropriate credit to the original author(s) and the source, provide a link to the Creative Commons license, and indicate if changes were made. The Creative Commons Public Domain Dedication waiver (http://creativecommons.org/publicdomain/zero/1.0/) applies to the data made available in this article, unless otherwise stated.

\section{References}


1. Chen Y, Nielsen J. Biobased organic acids production by metabolically engineered microorganisms. Curr Opin Biotechnol 2016; 37:165-172.

2. Sauer M, Porro D, Mattanovich D, Branduardi P. Microbial production of organic acids: expanding the markets. Trends Biotechnol 2008; 26:100-108.

3. Cok B, Tsiropoulos I, Roes AL, Patel MK. Succinic acid production derived from carbohydrates: An energy and greenhouse gas assessment of a platform chemical toward a bio-based economy. Biofuel Bioprod Bior 2014; 8:16-29.

4. Mukherjee V, Radecka D, Aerts G, Verstrepen KJ, Lievens B, Thevelein JM. Phenotypic landscape of non-conventional yeast species for different stress tolerance traits desirable in bioethanol fermentation. Biotechnol Biofuels 2017; 10:216.

5. Radecka D, Mukherjee V, Mateo RQ, Stojiljkovic M, Foulquie-Moreno MR, Thevelein JM. Looking beyond Saccharomyces: the potential of non-conventional yeast species for desirable traits in bioethanol fermentation. FEMS Yeast Res 2015; 15.

6. Xiao H, Shao ZY, Jiang Y, Dole S, Zhao HM. Exploiting Issatchenkia orientalis SD108 for succinic acid production. Microb Cell Fact 2014; 13.

7. Ahn JH, Jang YS, Lee SY. Production of succinic acid by metabolically engineered microorganisms. Curr Opin Biotechnol 2016; 42:54-66.

8. Park HJ, Bae JH, Ko HJ, Lee SH, Sung BH, Han JI, Sohn JH. Low-pH production of d-lactic acid using newly isolated acid tolerant yeast Pichia kudriavzevii NG7. Biotechnol Bioeng 2018; 115:2232-2242.

9. Toivari M, Vehkomaki ML, Nygard Y, Penttila M, Ruohonen L, Wiebe MG. Low pH D-xylonate production with Pichia kudriavzevii. Bioresour Technol 2013; 133:555-562.

10. Nugroho RH, Yoshikawa K, Shimizu H. Metabolomic analysis of acid stress response in Saccharomyces cerevisiae. J Biosci Bioeng 2015; 120:396-404.

11. Matsushika A, Negi K, Suzuki T, Goshima T, Hoshino T. Identification and Characterization of a Novel Issatchenkia orientalis GPI-Anchored Protein, loGas1, Required for Resistance to Low pH and Salt Stress. Plos One 2016; 11.

12. Soares EV. Flocculation in Saccharomyces cerevisiae: a review. J Appl Microbiol 2011; 110:1-18.

13. Bauer FF, Govender P, Bester MC. Yeast flocculation and its biotechnological relevance. Appl Microbiol Biotechnol 2010; 88:31-39.

14. Ceccato-Antonini SR. Biotechnological implications of filamentation in Saccharomyces cerevisiae. Biotechnol Lett 2008; 30:1151-1161.

15. Vallejo JA, Sanchez-Perez A, Martinez JP, Villa TG. Cell aggregations in yeasts and their applications. Appl Microbiol Biotechnol 2013; 97:2305-2318.

16. Vylkova S, Carman AJ, Danhof HA, Collette JR, Zhou H, Lorenz MC. The fungal pathogen Candida albicans autoinduces hyphal morphogenesis by raising extracellular $\mathrm{pH}$. mBio. doi:10.1128/mBio.00055-11. 
17. Ruiz-Herrera J, Sentandreu R. Different effectors of dimorphism in Yarrowia lipolytica. Arch Microbiol 2002; 178:477-483.

18. Douglass AP, Offei B, Braun-Galleani S, Coughlan AY, Martos AAR, Ortiz-Merino RA, Byrne KP, Wolfe $\mathrm{KH}$. Population genomics shows no distinction between pathogenic Candida krusei and environmental Pichia kudriavzevii: One species, four names. PLoS Pathog 2018; 14:e1007138.

19. Gong Z, Nielsen J, Zhou YJ. Engineering Robustness of Microbial Cell Factories. Biotechnol J 2017; doi.org/10.1002/biot.201700014.

20. Tan Z, Yoon JM, Nielsen DR, Shanks JV, Jarboe LR. Membrane engineering via trans unsaturated fatty acids production improves Escherichia coli robustness and production of biorenewables. Metab Eng 2016; 35:105-113.

21. Lin X, Qi Y, Yan D, Liu H, Chen X, Liu L. CgMED3 changes membrane sterol composition to help Candida glabrata tolerate low-pH stress. 2017; doi.org/10.1128/AEM.00972-17.

22. Turk M, Montiel V, Zigon D, Plemenitas A, Ramos J. Plasma membrane composition of Debaryomyces hansenii adapts to changes in pH and external salinity. Microbiology 2007; 153:35863592.

23. Y Q, H L, J Y, X C, L L. Med15B regulates acid stress response and tolerance in Candida glabrata. 2017; https://doi.org/10.1128/AEM.01128-17.

24. Goossens KV, lelasi FS, Nookaew I, Stals I, Alonso-Sarduy L, Daenen L, Van Mulders SE, Stassen C, van Eijsden RG, Siewers V, et al: Molecular mechanism of flocculation self-recognition in yeast and its role in mating and survival. MBio 2015; 6 .

25. Cullen PJ, Sprague GF, Jr. The regulation of filamentous growth in yeast. Genetics 2012; 190:23-49.

26. Zhang P, Du G, Zou H, Chen J, Xie G, Shi Z, Zhou J. Effects of three permeases on arginine utilization in Saccharomyces cerevisiae. Sci Rep 2016; 6:20910.

27. Kanjee U, Houry WA. Mechanisms of acid resistance in Escherichia coli. Annu Rev Microbiol 2013; 67:65-81.

28. Richard H, Foster JW. Escherichia coli glutamate- and arginine-dependent acid resistance systems increase internal pH and reverse transmembrane potential. J Bacteriol 2004; 186:6032-6041.

29. Xu S, Zhou J, Liu L, Chen J. Arginine: A novel compatible solute to protect Candida glabrata against hyperosmotic stress. Process Biochem 2011; 46:1230-1235.

30. Cheng Y, Du Z, Zhu H, Guo X, He X. Protective Effects of arginine on Saccharomyces cerevisiae against ethanol stress. Sci Rep 2016, 6:31311.

31. Zara G, Bou Zeidan M, Fancello F, Sanna ML, Mannazzu I, Budroni M, Zara S. The administration of L-cysteine and L-arginine inhibits biofilm formation in wild-type biofilm-forming yeast by modulating FLO11 gene expression. Appl Microbiol Biotechnol 2019; 103:7675-7685.

32. Braus GH, Grundmann O, Bruckner S, Mosch HU. Amino acid starvation and Gcn4p regulate adhesive growth and FLO11 gene expression in Saccharomyces cerevisiae. Mol Biol Cell 2003; 14:4272-4284. 
33. Tran VG, Cao M, Fatma Z, Song X, Zhao H. Development of a CRISPR/Cas9-Based Tool for Gene Deletion in Issatchenkia orientalis. mSphere 2019; doi.org/10.1128/mSphere.00345-19.

34. Conesa A, Gotz S, Garcia-Gomez JM, Terol J, Talon M, Robles M. Blast2GO: a universal tool for annotation, visualization and analysis in functional genomics research. Bioinformatics 2005 , 21:3674-3676.

35. Young MD, Wakefield MJ, Smyth GK, Oshlack A. Gene ontology analysis for RNA-seq: accounting for selection bias. 2010; doi:genomebiology.com/2010/11/2/R14

36. Mao X, Cai T, Olyarchuk JG, Wei L. Automated genome annotation and pathway identification using the KEGG Orthology (KO) as a controlled vocabulary. Bioinformatics 2005; 21:3787-3793.

37. Chin YW, Kang WK, Jang HW, Turner TL, Kim HJ. CAR1 deletion by CRISPR/Cas9 reduces formation of ethyl carbamate from ethanol fermentation by Saccharomyces cerevisiae. J Ind Microbiol Biotechnol 2016; 43:1517-1525.

38. Senouci-Rezkallah K, Schmitt P, Jobin MP. Amino acids improve acid tolerance and internal pH maintenance in Bacillus cereus ATCC14579 strain. Food Microbiol 2011; 28:364-372.

\section{Figures}

\section{Figure 1}

Effect of low-pH stress on the cell growth and morphology of P. kudriavzevii. (a) P. kudriavzevii JLY1107 and S. cerevisiae W303 were cultured in YPD medium at indicated pH conditions. (b) P. kudriavzevii were cultured in YPD or MM medium for $16 \mathrm{~h}$, and then collected into a tube, and then set aside for $2 \mathrm{~min}$ to observe flocculation. (c) Micrographs illustrating the morphology of P. kudriavzevii grown in YPD medium at different $\mathrm{pH}$ conditions. The photographs were taken from Leica DM3000. 
a

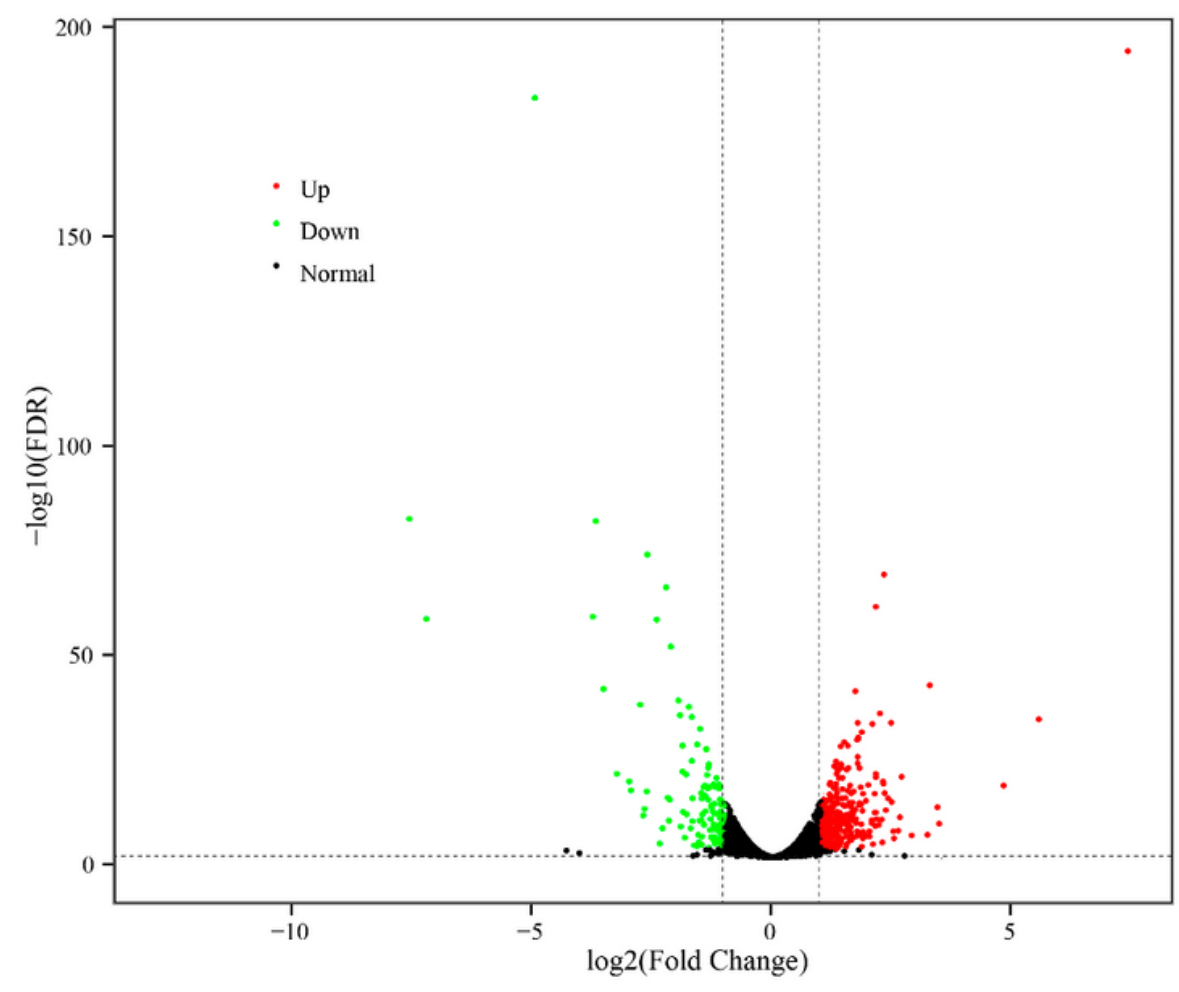

$\mathrm{b}$
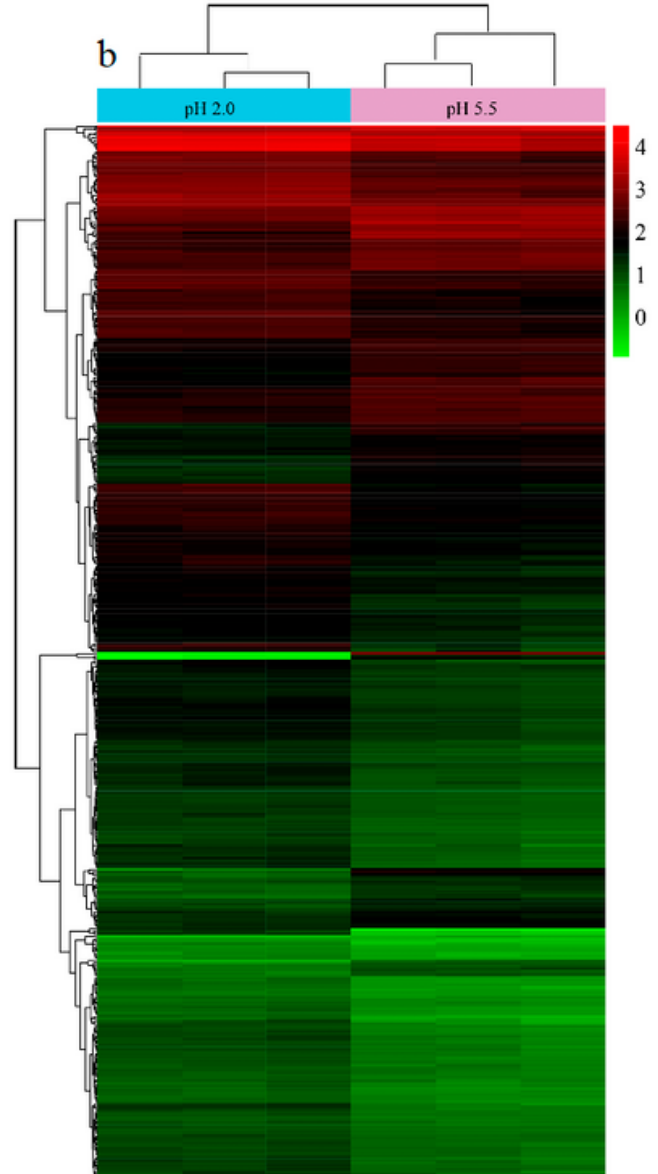

\section{Figure 2}

Expression profiles of differentially expressed genes (DEGs) in P. kudriavzevii incubated at different $\mathrm{pH}$ conditions. (a) Volcano plot showing DEGs. Imaginary lines indicate inclusion criteria for log2 fold change $\geq 1$ or $\leq-1$ and $-\log 10$ (FDR) $>2$. Red, up-regulated expressed genes in P. kudriavzevii incubated at $\mathrm{pH}$ 2.0; green, down-regulated expressed genes; black, genes does not meet inclusion criteria. (b) Hierarchical clustering of DEGs. Colors indicate expression levels of DEGs. 
a

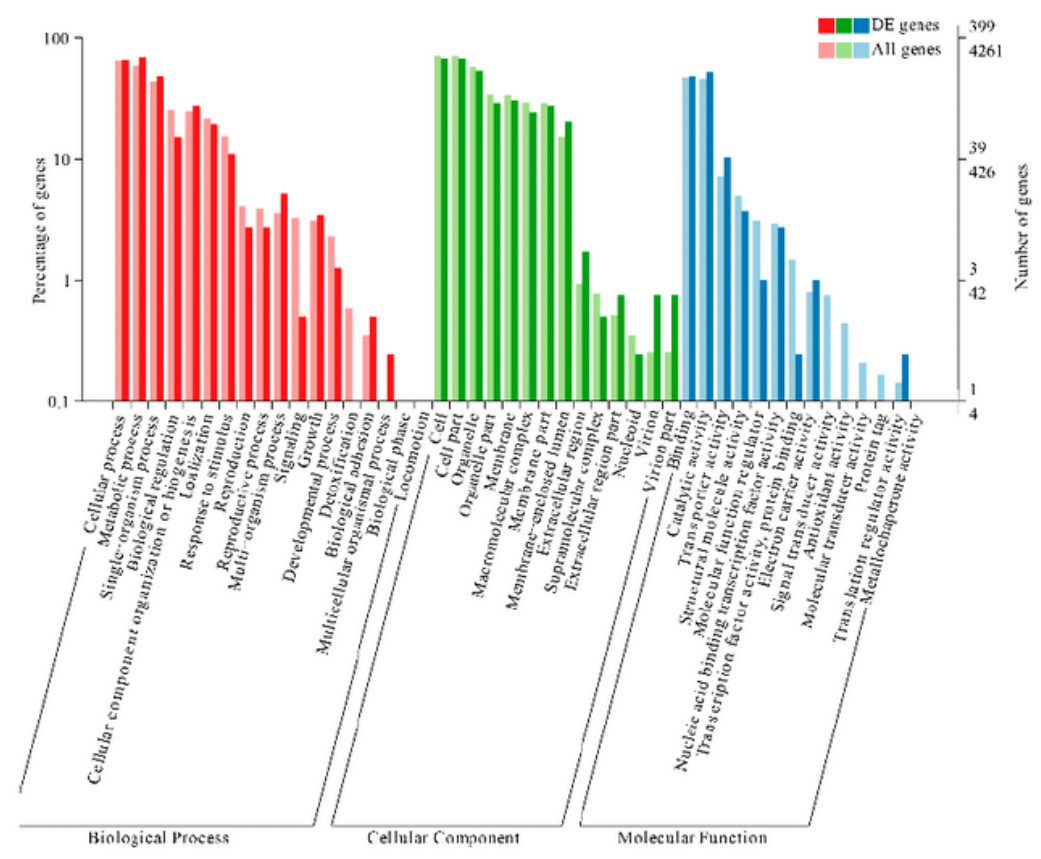

$\mathrm{b}$

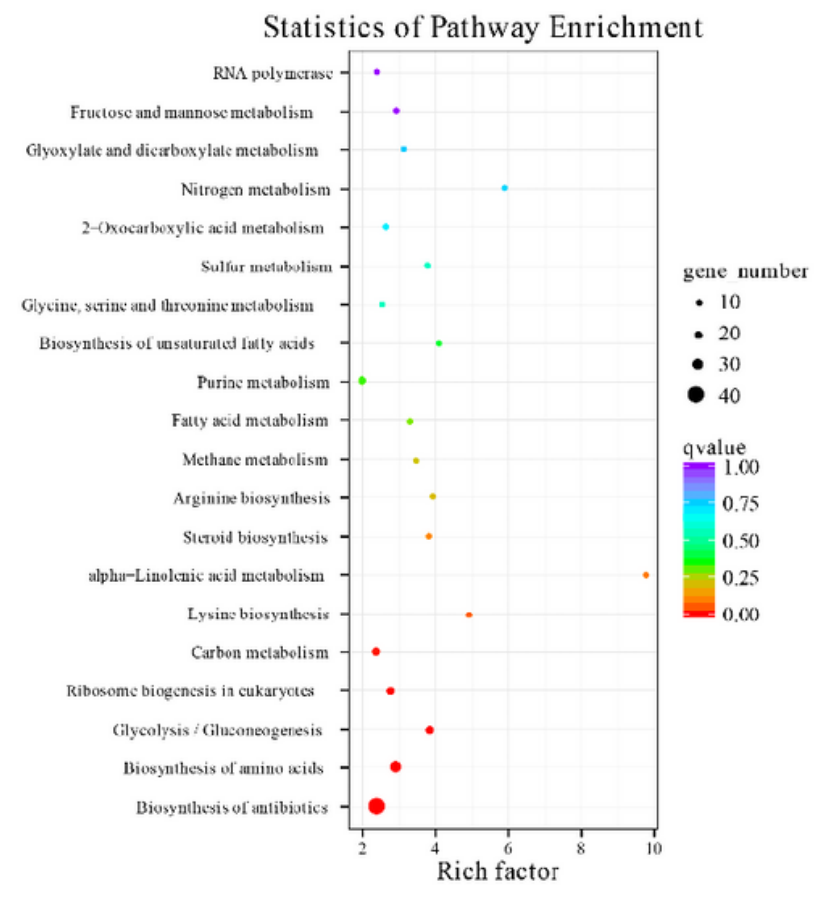

\section{Figure 3}

Gene Ontology (GO) and Kyoto Encyclopedia of Genes and Genomes (KEGG) pathway analyses. 
a



b

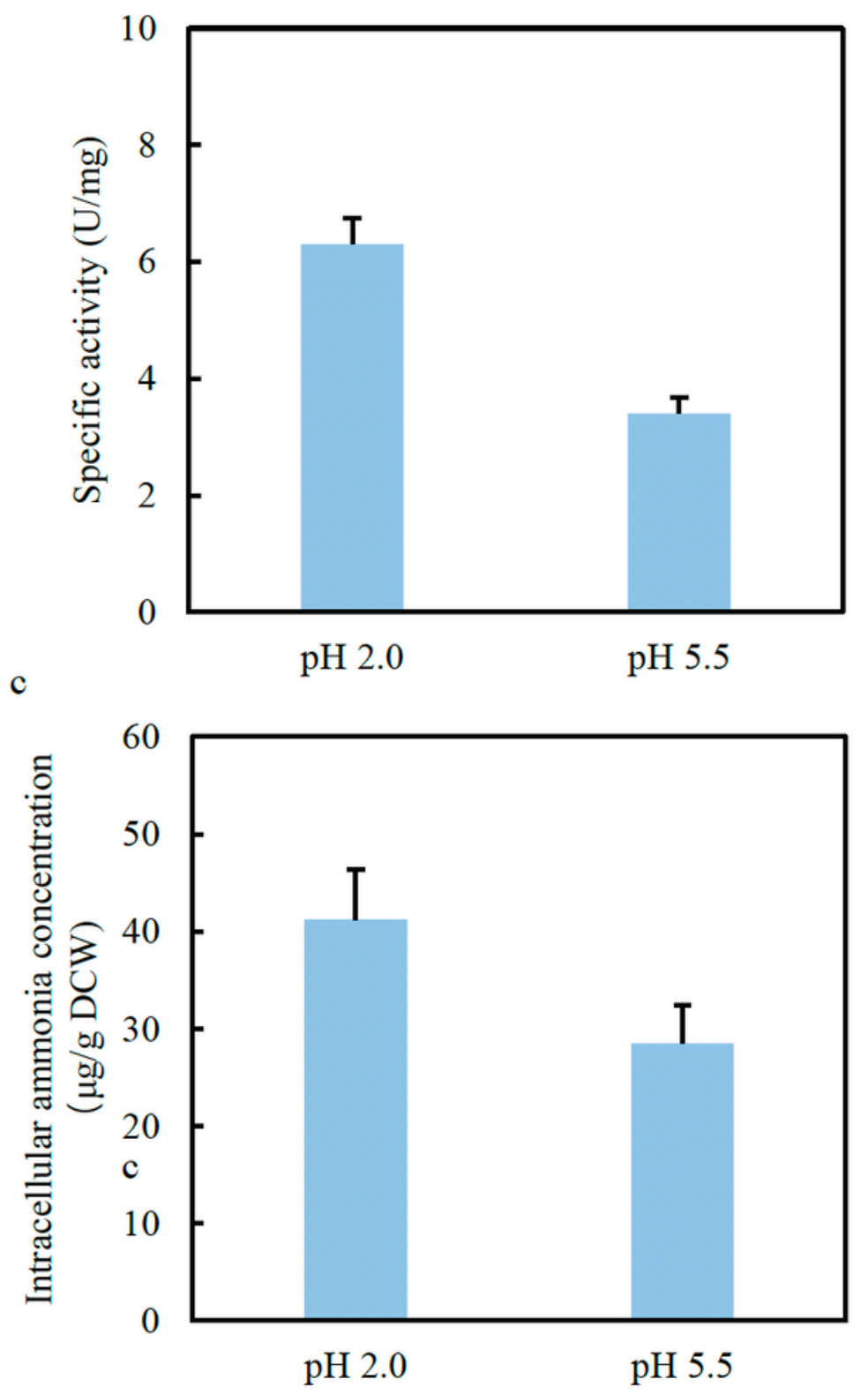

Figure 4

Low-pH stress induces arginine catabolism in P. kudriavzevii. (a) Differentially regulated genes involved in the arginine metabolism. Data represent fold changes in the transcription levels of the genes. Enzyme activities of arginase (b) and intracellular ammonia concentration of P. kudriavzevii was measured after cultured under $\mathrm{pH} 2.0$ and 5.5, respectively. All data from three independent replicates were used to calculate the mean and standard devuation. 
a
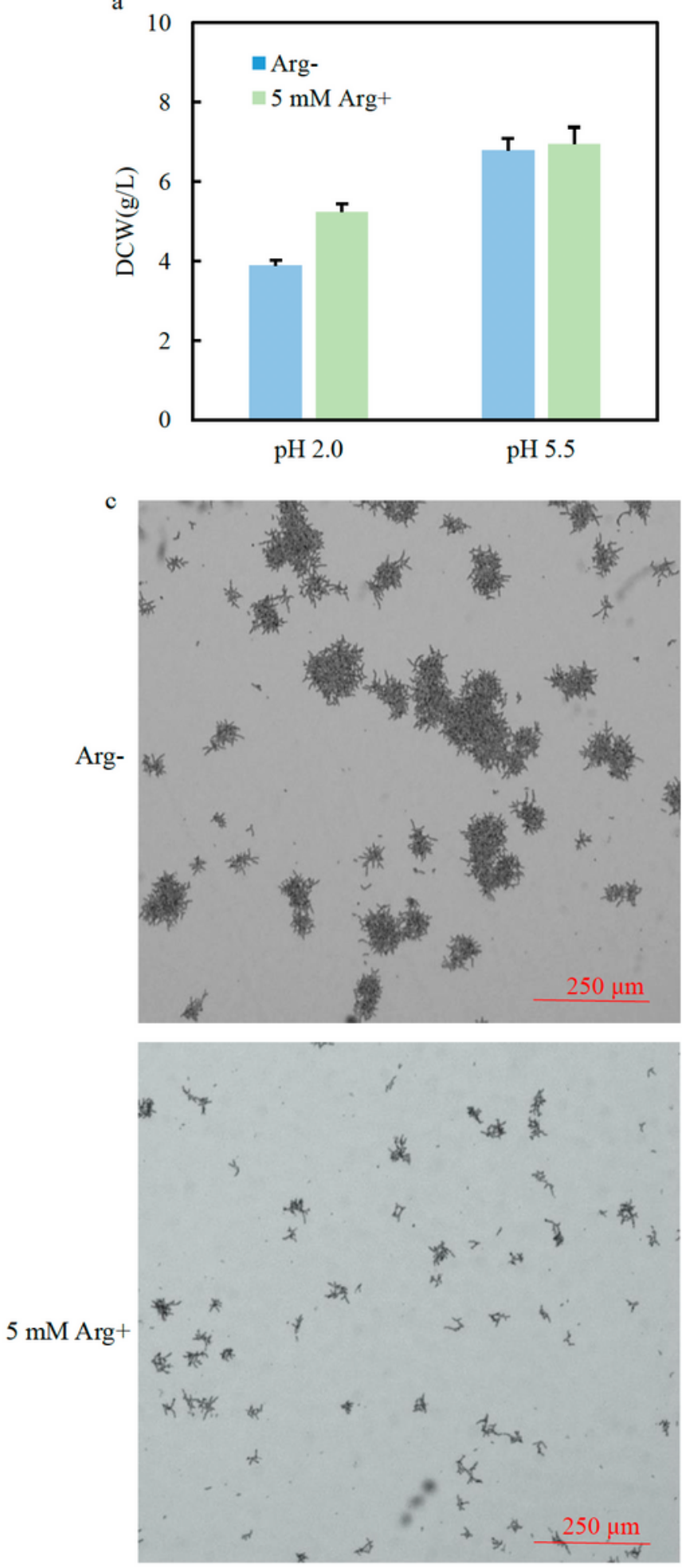

b
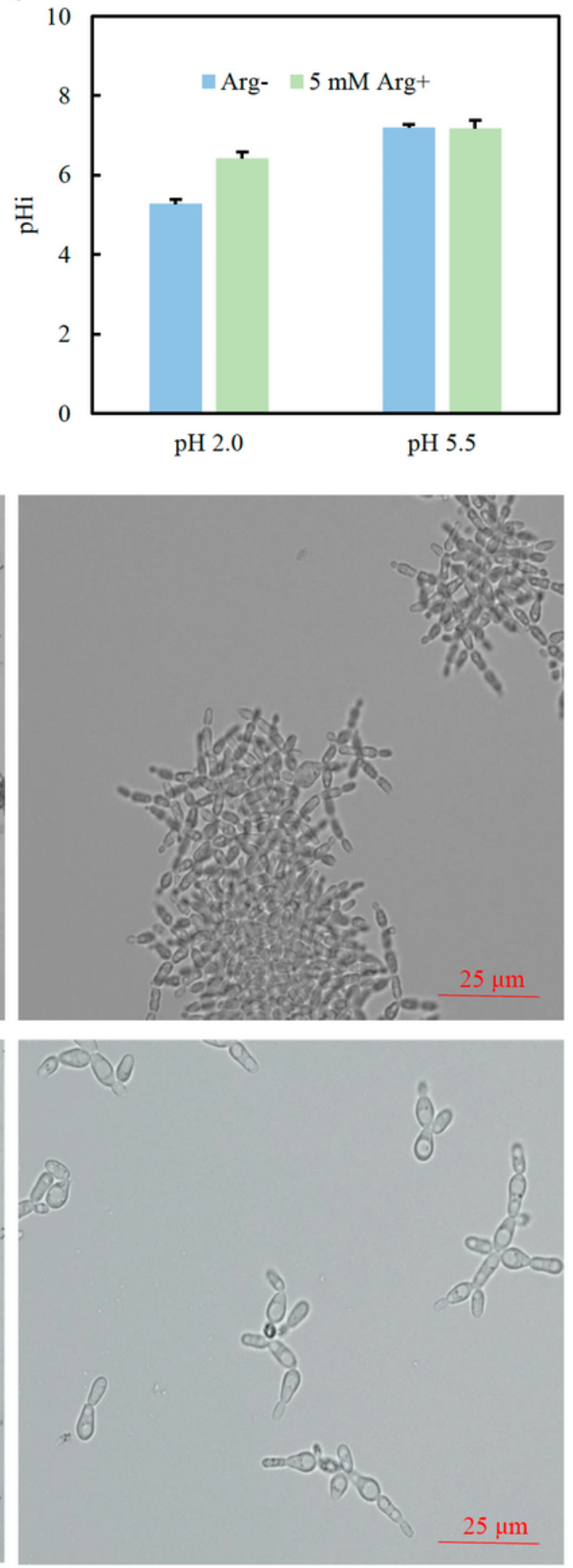

\section{Figure 5}

Effects of external addition of arginine on cell growth and morphological differentiation. DCW (a) and pHi (b) of P. kudriavzevii were measured in the culture with or without $5 \mathrm{mM}$ arginine application. All data from three independent replicates were used to calculate the mean and standard devuation. (c) Micrographs illustrating the morphology of P. kudriavzevii grown in YPD medium at pH 2.0 with or without $5 \mathrm{mM}$ arginine application. 


\section{Supplementary Files}

This is a list of supplementary files associated with this preprint. Click to download.

- AdditionalFile1.docx 